\title{
Developing Discriminatory Drug Dissolution Tests and Profiles: Some Thoughts for Consideration on the Concept and Its Interpretation
}

\author{
Saeed A. Qureshi, Ph.D.'
}

\begin{abstract}
Drug dissolution profiles are increasingly used to evaluate drug release characteristics of pharmaceutical products. Discriminatory dissolution profiles are highly desirable for differentiating between products having differences in pharmaceutical attributes (formulation and/or manufacturing processes differences) that may reflect corresponding differences in vivo. Commonly in the literature, differences in the profiles based only on pharmaceutical attributes have also been described as discriminatory. This appears to have created confusion in properly defining and developing discriminating profiles. This article should help to clarify definition/concept, independent of the apparatus or procedure used, so that proper discriminating profiles may be developed for improved evaluation of pharmaceutical products.
\end{abstract}

D rug dissolution (or release) testing is an analytical technique used to assess release profiles of drugs in pharmaceutical products, generally solid oral products such as tablets and capsules (1). This test gains its significance from the fact that if a drug from a product is to produce its effect, it must be released from the product and should generally be dissolved in the fluids of the gastrointestinal (GI) tract. Thus, a drug dissolution test may be considered as an indicator of potential drug release and absorption characteristics of a product in humans as well as in animals (2-6). Therefore, a dissolution test is often considered a surrogate for the assessment of availability of drugs in the body, generally termed bioavailability (7).

This link of dissolution (in vitro) to drug release in the body (in vivo) as commonly determined by bioavailability assessment is formally referred to as in vitro-in vivo correlation (IVIVC). This concept of IVIVC, in a quantitative and/or qualitative format, provides the basis for the assessment of quality of the products. Thus, the dissolution test is not only a procedure for product development but is also extensively used as a quality control technique because of this in vitro-in vivo association.

To reflect drug absorption behaviour in vivo or, more accurately drug release in vivo, drug dissolution tests are conducted in vitro, mimicking the physiological environment of the Gl tract. The Gl tract environment is represented by mild stirring of drug products in aqueous-based solutions, such as $0.1 \mathrm{~N} \mathrm{HCl}$ or buffers having $\mathrm{pH}$ values in the range of 4-7.5. The cumulative percentage of a drug dissolved at a number of time points is determined and may be reported as a plot of $\%$ drug dissolved versus sampling times. The resulting graph is commonly referred to as a

${ }^{1}$ Bureau of Pharmaceutical Sciences, Therapeutic Products Directorate, Health Products and Food Branch, Health Canada,Ottawa, Canada K1A OL2 "dissolution profile" and provides a means of comparison with in vivo drug release to establish absorption characteristics of drugs from products in humans $(6,8-9)$.

Further, because of the above-mentioned in vitro-in vivo association, it is generally considered that if a dissolution profile of a test product matches that of a reference product, then the test product should behave similarly to the reference product in vivo (i.e., both will have similar bioavailabilities and will be considered as bioequivalent). Conversely, if the profile of the test product is different from that of the reference product, then the test product might behave differently in humans.

This practice of describing dissolution results or profiles is commonly referred to as providing discriminating dissolution profiles, and the test as a discriminatory test. That is, a dissolution test is expected to discriminate whether dissimilar products are from different manufacturing batches of the same product or from different products such as generics. It is most important to note that the terminology of "discrimination" should be related to and based on similarity or dissimilarity of in vitro results to in vivo results only. It is further important to note that a test should only be considered discriminatory if dissolution profiles obtained are dissimilar for dissimilar in vivo profiles (i.e., products should be bioinequivalent).

If different dissolution profiles are obtained for products with the same or different formulations or manufacturing attributes but with similar in vivo characteristics, they may not be considered as discriminating profiles, and the test that produces such profiles should not be considered as a discriminatory test. The differences in profiles for products having similar release characteristics in vivo (i.e., for bioequivalent products) should be considered as an expected and acceptable variation in dissolution results from acceptable products without any negative therapeutic consequences. Such differences in dissolution profiles should 

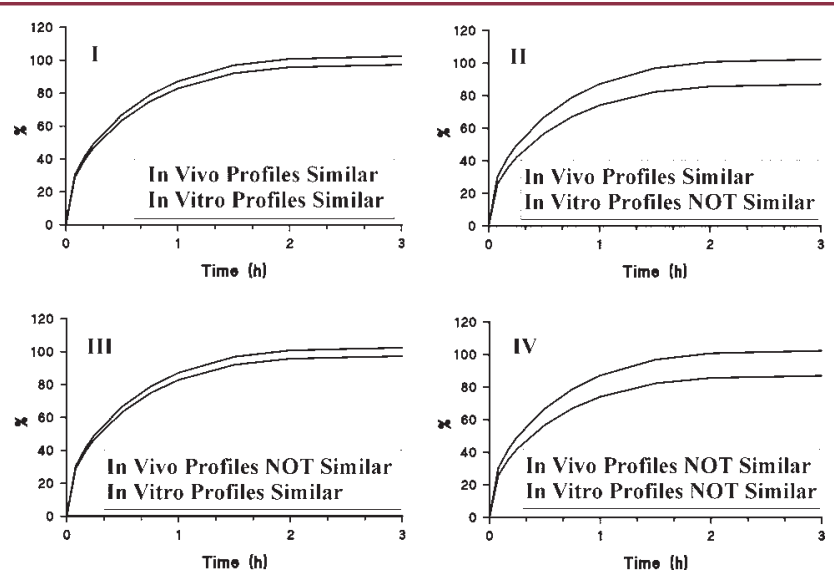

Figure 1. Possible testing outcome scenarios, as drug dissolution (release) profiles, of products with in vivo similar (bioequivalent) and dissimilar (bioinequivalent) release characteristics.

form the basis of setting tolerances for quality control purposes for acceptable products and not for establishing discrimination or differences.

However, there appears to be some confusion in the literature regarding the term "discriminating profiles," which is used for profiles from both bioinequivalent and bioequivalent products (10-11). That is, differences in profiles based purely on differences due to some manufacturing or formulation attributes without any reference to in vivo release characteristics are often referred to as discriminatory profiles. This may not be an accurate interpretation of the concept of discriminating profiles.

This article will present a discussion to help in clarifying this confusion, so that a common and appropriate terminology may be applied that will help in improved product development and assessment.

It is to be noted that the discussion presented here relates to the concept of developing and defining discriminatory dissolution tests irrespective of the testing technique employed. Examples are provided to highlight the concept rather than to discuss a particular technique. Furthermore, comparisons described should not be considered as comparison of testing using different types of spindles, but testing with two different testing environments created by the use of different spindles. For appropriate dissolution testing, it is the environment that is important, not the choice of the spindle type.

This concept may be explained by considering a situation where two products $A$ and $B$ are compared in vivo to a reference product $(R)$ resulting in one being found bioequivalent (say product $A$ ) and the other not bioequivalent (say product B). It is important to note that both products (bioequivalent or not) have differences in their formulation and/or manufacturing attributes compared to the reference product. Furthermore, dissolution testing of these products is conducted using a standard dissolution procedure. For example, suppose dissolution experiments were conducted using USP Paddle Apparatus at $75 \mathrm{rpm}$ using water as a dissolution medium containing some solublizing agent (e.g., $0.5 \%$ sodium lauryl sulphate).

The possible outcomes of this dissolution study would be as follows: (I) Bioequivalent products ( $A$ and $R$ ) give similar dissolution profiles. (II) Bioequivalent products ( $A$ and $\mathrm{R}$ ) give dissimilar dissolution profiles. (III) Bioinequivalent products (B and R) give similar dissolution profiles. (IV)

Bioinequivalent products ( $B$ and $R$ ) give dissimilar profiles.

These outcomes are simulated as profiles in Figure 1. For similar and dissimilar profiles, a difference of less than 10\% and greater than $10 \%$ drug release at each sampling time, respectively, may normally be used for $\mathrm{f} 2$ similarity factor criteria (9). In this particular example, the lower profiles represent those of the test products that show 5 and $15 \%$ lower drug dissolution than the reference product for similar and dissimilar profiles, respectively.

From the discriminating profiles perspective, two of the four outcomes would be considered accurate (i.e., I and IV), as these profiles correctly reflect the in vivo behaviour of the products. However, only profiles in outcome IV should be considered discriminating, and the test should be considered as a discriminatory test. Note that in both cases (I and IV), profiles are different, reflecting differences in products attributes, but only outcome IV will be considered as discriminating profiles as it differentiates profiles based on the in vivo outcome. Outcome l, through having different profiles, would be considered as similar and may be used for setting tolerances for acceptable products.

Cases II and III represent inaccurate outcomes of the dissolution testing. Commonly, such situations are described in the literature as over- and under-discriminating profiles or tests (12-13). However, these may not be considered discriminating profiles or tests; in fact, they are inappropriate tests resulting in the false characterization of products. A corresponding analogy for a bioequivalency test would be that products are declared either bioequivalent or not. There are no "under" or "over" bioequivalent products. Similarly, a dissolution test has to be discriminatory or not. There should not be an over- or under-discriminatory test. A discriminatory test will yield similar dissolution profiles, which may or may not be overlapping, for bioequivalent products and different profiles for bioinequivalent products.

When all the curves shown in the Figure 1 are observed in isolation (i.e., without a reference to their bioequivalency characteristics), curves within all sets are different from each 

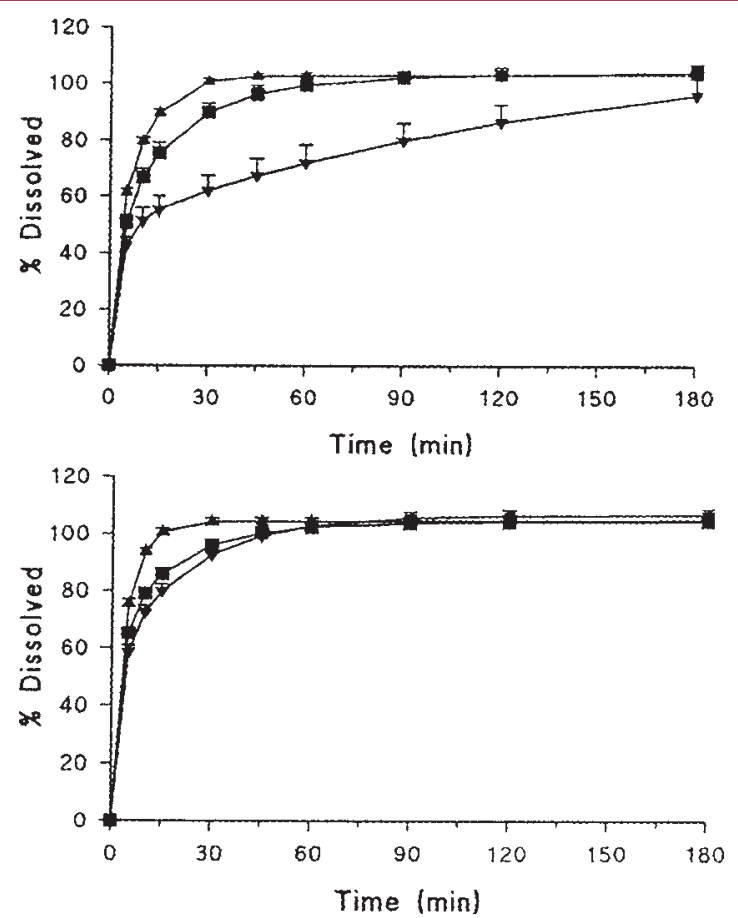

Figure 2. Percent drug release profiles of three immediate-release carbamazepine tablet products $(200 \mathrm{mg}$ ) using the USP paddle spindle (top) and crescent-shaped spindle (bottom). Experiments were conducted using spindle rotation speed of $75 \mathrm{rpm}$ with $900 \mathrm{~mL}$ water containing 1\% sodium lauryl sulphate (22).

other, as might be expected since each set represents a different set of products (or perhaps different batches). However, not all these curves can be considered as discriminating, since for the appropriate definition/concept of discrimination, one requires a reference of acceptable or unacceptable outcome. In our case, reference to acceptable or unacceptable outcomes refers to drug release in humans. Therefore, the test becomes discriminatory, and profiles as in case IV become discriminating as these relate to reference accurately. On the other hand, tests that resulted in cases II and III are not discriminatory, and neither are the profiles.

In short, a dissolution test may result in two sets of outcomes, one in which the test may be considered as discriminatory and others in which it is not. It is only the in vivo characteristics that will dictate discriminating ability of the test or the testing environment.

As all the above-mentioned outcomes are based on the same experimental conditions, an obvious question would be, is this test accurate in providing the true outcome and, if so, which set of outcomes is accurately represented by the tests? Assuming the testing approach used in this example is reflecting (simulating) in vivo accurately, then one has to question why the sets of profiles (II and III) are not providing an accurate picture. However, if sets II and III indicate a mismatch with the in vivo environment of the testing system, then obviously profiles in sets I and IV cannot be relied upon as well, since the results are obtained from an inappropriate test.

The impact or relevance of an environment or technique may be evaluated if the results are obtained and compared using another environment, and the outcomes (dissolution results) evaluated based on some reference, which in this case is in vivo release. This concept is explained by discussing the results obtained in studies reported in the literature.

Three interchangeable, thus bioequivalent, commercially available IR carbamazepine tablet products $(200 \mathrm{mg}$ ) were analysed in two different environments. One was created using USP paddle spindle as described in the USP monograph, and the other using a newly suggested crescentshaped spindle. The resulting profiles from these tests are shown in Figure 2.

As expected, there are two sets of outcomes depending on the testing environment employed. One set shows larger differences in dissolution profiles among products. If one sees these results in isolation, the larger the differences between profiles, the better the testing environment is considered as it is differentiating/discriminating the product characteristics better. However, the critical thing to consider here is which "product characteristics" are referred to? As stated in the beginning of the article and described extensively in the literature, the "product characteristics" referred to or evaluated using dissolution testing is in vivo drug release. Therefore, an appropriate testing environment will be the one that would provide better reflection of in vivo drug release characteristics. As the tested products are bioequivalent from an in vivo perspective, a dissolution environment that shows minimal differences and does not discriminate among the products should be considered the appropriate one. Therefore, in this case, testing with USP paddle may not be considered as discriminatory, but this is, in fact, an inaccurate reflection of the testing environment and the products characteristics.

Therefore, when considering a discriminatory dissolution test, the test must have a link and relevance to in vivo environment and be capable of reflecting appropriate in vivo release characteristics. In the absence of such an in vivo link, testing would be of limited value. The environment created by the use of the crescent-shape spindle appears to provide this link.

It is usually argued that, using the paddle spindle, dissolution testing may not reflect accurate physiological environment, which results in the poor predictability of in vivo dissolution behaviour of products. Then, to make the tests more physiologically appropriate, the tests conditions may 
be adjusted in retrospect, so that dissolution test results may correspond to in vivo outcome (14-16). Thus, in practice, the experimental conditions, including the use of different apparatus, are adjusted to achieve the desired outcome. This is the currently recommended approach for conducting dissolution testing and is widely practised $(6,15)$. However, it appears that it is based on a flawed logic in that it seeks a desired outcome rather than an evaluation of actual drug release characteristics of the test products. By its nature, such product-specific tests become non-discriminatory tests and may not be useful for comparative testing of products (different batches or products). Therefore, in such cases, the currently used approach for product-specific dissolution testing may not be considered as being able to provide discriminatory tests.

It is most critical that one should be clear about differences in IVIVM (in vitro-in vivo matching) and IVIVC (in vitro-in vivo correlation), which appears to be causing great confusion in dissolution testing practice. The objective of dissolution testing is to achieve predictability of testing based on (co)relationship, that is, differences/similarity in vitro should be reflected in vivo and vice versa under the same testing conditions/environment whether products are from same lot, different formulation, or different products. On the other hand, if dissolution testing does not provide the expected predictability, and one is required to change the experimental conditions from product to product to obtain the desired in vitro response, in particular after the fact, then this should be considered as matching of the bioresults or IVIVM. This IVIVM does not provide any predictability of results, and thus is of limited use for determining quality of a test product. Often, these two concepts (IVIVM versus IVIVC) are interchangeably used causing great confusion. However, for proper dissolution testing and for developing appropriate IVIVC, a common and product-independent method is an essential requirement.

Such product-dependent IVIVM environments are commonly reported for evaluating ER products. Often for these products, not only testing environments employed are different than used for IR products, but even within ER category, testing is conducted using different environments.

This approach of using a product-dependent multi-environment would not be considered as bio-relevant, as the in vivo environment remains the same whether product is of $I R$ or ER type. Therefore, an appropriate comparison of release characteristics of IR and ER products, including different variations within each category, can only be made if the testing environment is the same.

It is also to be noted that, if suggested, one may test IR and ER products by different dissolutions methods because of the difference in the nature or behaviour of the formulations

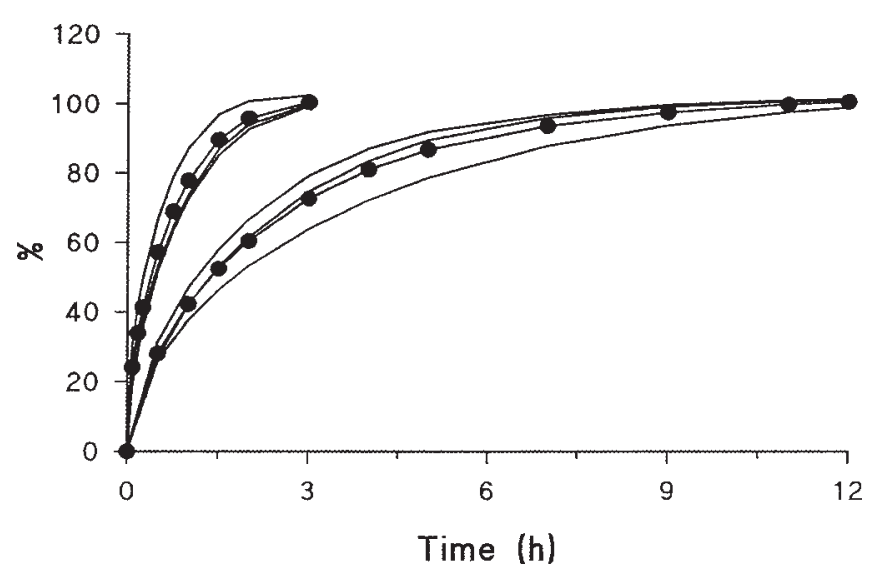

Figure 3. Percent drug release profiles of three immediate (top) and three extended-release (bottom) carbamazepine tablet products $(200 \mathrm{mg})$ using crescent-shaped spindle. (- -) and (- - ) represent drug release values as means for individual products and grand mean from all products, respectively, from the respective set of immediate- and extended-release products. Experiments were conducted using spindle rotation speed of 25 rpm with $900 \mathrm{~mL}$ water containing $0.5 \%$ sodium lauryl sulphate (24).

or manufacturing attributes, then, by its nature the method becomes non-discriminatory. A discriminatory dissolution method, by its very assumption, is required to differentiate between products based on formulation or manufacturing attributes. If the technique or method is not able to differentiate between release from IR and ER products, which represent wider differences, then one would not anticipate that such a method could differentiate smaller differences as one would anticipate within a product category or their batches.

A more appropriate testing environment would be one in which IR and ER products would be analysed under the same environment and drug release clearly differentiated between these two product types. There are reports in the literature that indicate that an improved and comparable in vitro-in vivo environment may be obtained if stirring and mixing behaviour relates better to the physiological environment (17-21). Therefore, if one creates a more appropriate and relevant mixing and stirring environment in vitro, dissolution testing may be more appropriate (i.e., one should be able to test multiple products using the same experimental condition as in vivo dictates).

Recently, it has been suggested that a more appropriate environment may be created by altering the mixing and stirring mechanism within dissolution vessel using a new spindle (crescent-shaped) (22-24). Obviously, if such an environment is more appropriate, then it should address the issues described above, that is, IR and ER products should be able to analysed using the same environment and drug release should be clearly different between these two categories. 
Let us consider the comparison of drug release characteristics between 200-mg strength immediate-release (IR) and extended-release (ER) carbamazepine tablets products (24). It should be noted that these products are interchangeable and thus bioequivalent within product types (i.e., IR and ER). Obviously, these products types cannot be considered equivalent to each other, as they are shown to have very different in vivo release characteristics, IR versus ER. A suitable dissolution method would be one that reflects such differences in behaviour.

If one is to analyse these products using the methodology described in the USP, the observed results for the products cannot be compared, as test conditions are product dependent (i.e., different methodologies are suggested for IR and ER products) (25-27). One would not be able to ascertain whether observed differences in drug release are due to the products or experimental conditions. However, for the purposes of a discriminatory test, the test condition and procedure have to be the same rather than as described in respective carbamazepine products USP monographs.

On the other hand, drug release profiles for these products, as shown in Figure 3, can be compared as these are obtained using the same experimental conditions (i.e., crescent-shaped spindle at $25 \mathrm{rpm}$ using $0.5 \% \mathrm{SLS}$ in water). Since the experimental conditions are the same, observed results would be reflective of product characteristics and can be compared within and between product classes. Clearly, as one would anticipate, the profiles fall into two distinct groups, one reflecting IR and the other ER products. The method clearly discriminates between two categories of products based on their expected in vivo characteristics, but also shows similarity, with slight differences in profiles, within the respective group reflecting differences in formulation and manufacturing attributes in each group. Not only does the method clearly differentiate products in each group, but it also provides a true reflection of the bioavailability/ bioequivalence behaviour of the product. Therefore, such an environment or a test may be considered as reflecting appropriate in vivo environment with discriminating ability.

Thus, for a dissolution test to be discriminatory, products must be analysed using the same experimental conditions, and the profiles (results) should reflect appropriate in vivo behaviour of the products (i.e., bioequivalent products should show similar profiles, and bioinequivalent product should give dissimilar profiles). If dissolution results do not correspond to in vivo characteristics, then the cause of this discrepancy should be explored (e.g., interaction between active and inactive ingredients, unusual in vivo behaviour such as precipitation, degradation) rather than trying to obtain the desired outcome (IVIVM) by changing the experimental conditions specific to the test product or its type (IR or ER). As described earlier, a product-specific test developed to match the in vivo results should not be considered a discriminatory test, and such practice may result in inappropriate interpretation of results and characterization of products.

In short, for a dissolution test to be discriminatory, the test should reflect differences in the products characteristics reflective of their in vivo release (bioavailability) characteristics. Differences in dissolution profiles based on differences in formulation and manufacturing attributes alone, in particular for bioequivalent products, may not constitute discriminating dissolution profiles but normal expected variation in results related to product differences. As the commonly used USP paddle spindle dissolution methods do not provide an appropriate stirring and mixing environment reflective of in vivo conditions, it is unlikely that tests using such methodology would provide bio-relevant, hence discriminatory, dissolution testing. Further, results would be of limited value and perhaps could be misleading. For improved dissolution testing, in particular for discriminatory tests, better stirring and mixing environments in the dissolution vessels appear to be needed.

\section{REFERENCES}

1. USP General Chapter on Dissolution $<711>$. United States Pharmacopeia and National Formulary; United States Pharmacopeial Convention, Inc.: Rockville, MD, 2005; pp 2412-2414.

2. Levy, G. Effect of dissolution rate on absorption, metabolism, and pharmacologic activity of drugs. J. Mond. Pharm. 1967, 3, 237-254.

3. Wagner, J. G. Biopharmaceutics and Relevant Pharmacokinetics; Drug Intelligence Publications: Hamilton, IL, 1971; Chapter 10.

4. Abdou, H. M. Dissolution, Bioavailability, and Bioequivalence; Mack Publishing Company: Easton, PA, 1989; 6.

5. Dissolution Testing of Immediate Release Solid Oral Dosage Forms; Guidance for Industry; U.S. Department of Health and Human Services, Food and Drug Administration, Center for Drug Evaluation and Research (CDER), U.S. Government Printing Office: Washington, DC, 1997. http://www.fda.gov/cder/guidance/1713bp1.pdf

6. Extended Release Oral Dosage Forms: Development, Evaluation, and Application of In Vitro/In Vivo Correlations; Guidance for Industry; U.S. Department of Health and Human Services, Food and Drug Administration, Center for Drug Evaluation and Research (CDER), U.S. Government Printing Office:Washington, DC, 1997. http://www.fda.gov/cder/guidance/1306fnl.pdf. 
7. Waiver of In Vivo Bioavailability and Bioequivalence Studies for Immediate Release Solid Oral Forms Containing Certain Active Moieties/Active Ingredients Based on Biopharmaceutics Classification System; Guidance for Industry; U.S. Department of Health and Human Services, Food and Drug Administration, Center for Drug Evaluation and Research (CDER), U.S. Government Printing Office:Washington, DC, 2000. http://www.fda.gov/cder/guidance/3618fnl.pdf.

8. Moore, J. J.; Flanner, H. H. Mathematical comparison of curves with an emphasis on in vitro dissolution profiles. Pharm. Tech. 1996, 20,64-74.

9. Shah, V. P., Tsong, L., Sathe, P., Williams, R. L. Dissolution Profile Comparison Using Similarity Factor, $f_{2}$. Dissolution Technol. 1999, 6 (3), 21.

10. USP General Chapter $<1092>$ the Dissolution Procedure: Development and Validation. Pharm. Forum 2004, 30, 351-363.

11. USP General Chapter on In Vitro and In Vivo Evaluation of Dosage Forms $<1088>$ ). United States Pharmacopeia and National Formulary; United States Pharmacopeial Convention, Inc.: Rockville, MD, 2005, pp 2636-2641.

12. McNamara, P. J.; Foster, T. S.; Digenis, G. A.; Patel, R. B.; Craig, W. A.; Welling, P. G.; Rapaka, R. S.; Prasad, V. K.; Shah, V. P. Influence of tablet dissolution on furosemide bioavailability: a bioequivalence study. Pharm. Res. 1987, 4, 150-153.

13. Meyer, M. C.; Straughn, A. B.; Mhatre, R. M.; Shah, V. P.; Williams, R. L.; Lesko, L. J. Lack of in vivo/in vitro correlations for $50 \mathrm{mg}$ and $250 \mathrm{mg}$ primidone tablets. Pharm Res. 1998, 15, 1085-1089.

14. Lake, O. A.; Olling, M.; Barends, D. M. In vitro/in vivo correlations of dissolution data of carbamazepine immediate release tablets with pharmacokinetic data obtained in health volunteers. Eur. J. Pharm. Biopharm. 1999, 48, 13-19.

15. Leeson, L. J. In vitro/in vivo correlations. Drug Inf.J. 1995, 29, 903-915.

16. Abrahamsson, B.; Johansson, D.; Torstensson, A.; Wingstrand, K. Evaluation of solubilizers in the drug release testing of hydrophilic matrix extended- release tablets of felodipine. Pharm. Res. 1994, 8 , 1093-1097.

17. Cox, D. C.; Wells, C. E.; Furman, W. B.; Savage, T. S.; King, A. C. Systematic Error Associated with Apparatus 2 of the USP Dissolution Test II: Effects of Deviations in Vessel Curvature From That of a Sphere. J.Pharm. Sciences. 1982, 71, 395- 399.

18. Kukura, J.; Baxter, J. L.; Muzzio, F. J. Shear Distribution and Variability in the USP Apparatus 2 under Turbulent Conditions. Int. J. Pharm. 2004, 26, 9-17.

19. McCarthy, L. G.; Kosiol, C.; Healy, A. M.; Bradley, G.; Sexton, J. C.; Corrigan, O. I. Simulating the Hydrodynamic Conditions in the United States Pharmacopeia Paddle Dissolution Apparatus. AAPS PharmSciTech 2003, 4, 172-187.

20. Morihara, M.; Aoyagi, N.; Kaniwa, N.; Katori, N.; Kojim, S. Hydrodynamic Flows Around Tablets in Different Pharmacopeial Dissolution Tests. Drug Develop. Ind. Pharm. 2002, 28, 655-662.

21. Qureshi, S. A.; Shabnam, J. Cause of high variability in drug dissolution testing and its impact on setting tolerance. Eur.J. Pharm. Sci. 2001, 12, 271-276.

22. Qureshi, S. A.; Shabnam, J. Application of a new device (spindle) for improved characterization of drug release (dissolution) of pharmaceutical products. Eur.J. Pharm. Sci. 2003, 19, 291-297.

23. Qureshi, S.A. A new crescent-shaped spindle for drug dissolution testing-but why a new spindle? Dissolution Technol. 2004, 11 (4) 13-18.

24. Qureshi, S. A. Choice of rotation speed (rpm) for biorelevant drug dissolution testing using a Crescentshape spindle. Eur.J.Pharm. Sci. 2004, 23, 271-275.

25. Leeson, L. J.; Mayersohn, M. Letter to the Editor. Dissolution Technol. 2005, 12, 24-33.

26. Carbamazepine Tablets Monograph. United States Pharmacopeia and National Formulary; United States Pharmacopeial Convention, Inc.: Rockville, MD, 2005; pp 342-343.

27. Carbamazepine Extended-Release Tablets Monograph. United States Pharmacopeia and National Formulary; United States Pharmacopeial Convention, Inc.: Rockville, MD, 2005; pp 343-344. 